\title{
Digital Baseband Compensation of I/Q Imbalance in Mobile OFDM
}

\author{
Balachander Narasimhan, Sili Lu, Naofal Al-Dhahir and Hlaing Minn \\ Department of Electrical Engineering, University of Texas at Dallas \\ Email: \{bxn062000, sx1059000, aldhahir, hlaing.minn\}@utdallas.edu
}

\begin{abstract}
I/Q imbalance and high mobility in OFDM systems result in performance-limiting intercarrier interference (ICI). However, the nature of ICI due to each of them is quite different. Unlike previous works which considered these two impairments separately, we develop a unified mathematical framework to characterize and mitigate ICI when both impairments are present. In addition, we exploit the special ICI structure to design efficient OFDM channel estimation and digital baseband compensation schemes for I/Q imbalance under high-mobility conditions.
\end{abstract}

\section{INTRODUCTION}

Next-generation broadband wireless systems are required to provide higher rates, better reliability, and higher user mobility while targeting lower cost, lower power consumption, and higher levels of integration (smaller form factor). Orthogonal Frequency Division Multiplexing (OFDM) has been adopted as the PHY technology of choice for most broadband wireless standards (such as IEEE 802.16 and IEEE 802.11). To support higher data rates, the future trend is to operate at higher carrier frequencies and use higher-order signal constellations (e.g. 64 QAM) which are more sensitive to implementation imperfections such as I/Q imbalance and to mobility.

I/Q Imbalance refers to amplitude and phase mismatches between the in-phase (I) and quadrature (Q) branches. In the ideal case, these branches should have equal amplitude and $90^{\circ}$ phase shift but this is not the case in practice. Mobility (Doppler effect) destroys sub-carrier orthogonality within each OFDM symbol by introducing ICI and becomes more severe at higher speeds, higher carrier frequencies, and for larger OFDM block durations (necessary to combat severe channel frequency selectivity). Effective compensation of these impairments and reliable coherent signal detection require accurate channel estimates at the receiver in the presence of these impairments. This is a very challenging task especially for broadband channels under high mobility (due to the increased number of channel parameters to be estimated and due to fast channel time variations).

I/Q imbalance is especially pronounced in direct-conversion receivers and like other $\mathrm{RF}$ impairments in the analog components, it is exacerbated due to fabrication process variations which are difficult to predict or control, increase with the downscaling of fabrication technologies, and cannot be efficiently or

This work was supported in part by the National Science Foundation (NSF) under contract CCF 04-30654 and by a gift from Texas Instruments Inc. completely canceled in the analog domain due to power-areacost tradeoffs. New paradigms like "Dirty RF" [1], to achieve lower costs and power, deliberately relax the tolerances of analog front end assuming baseband compensation techniques. All of the above-mentioned considerations coupled with the fact that embedded digital processors and custom ASICs in mobile devices are becoming more powerful, motivate this research which aims at developing high-performance lowcomplexity digital baseband compensation techniques for I/Q imbalance in mobile OFDM systems.

Our proposed research is distinct from previous research in this area (such as [3]- [8]) in the following two key aspects. Previous works in this area consider either I/Q imbalance or mobility. However, in a broadband wireless systems, it is likely that both impairments are present. We develop a generalized mathematical model to quantify the joint ICI effects of both impairments in Section II. This will allow us to evaluate the individual as well as the combined effects of these impairments on system performance, better understand their interactions, and identify the dominant impairment(s) under a specific operating scenario. Furthermore, we will exploit the channel and ICI structure to reduce the complexity of digital baseband compensation algorithms.

This paper is organized as follows. In Section II, we present ICI mathematical models in the presence of I/Q imbalance only, mobility only, and both. Section III proposes a lowcomplexity digital baseband compensation scheme to mitigate their effects. In Section IV, we describe effective algorithms to estimate the overall channel response in the presence of these two impairments. Simulation results are given in Section VI and the paper is concluded in Section VII.

Notation: Boldface capital letter (A) denotes matrix and boldface small letter (a) denotes a vector. $(\cdot)^{H}$ denotes the Hermitian (conjugate transpose) of a matrix or vector and $(\cdot)^{*}$ denotes the conjugate of a matrix, vector or scalar. $(\cdot)^{T}$ denotes the transpose of a matrix or vector. $N$ is the size of Discrete Fourier Transform (DFT) of the OFDM system which is a power of $2 . \mathbf{F}$ is the unitary DFT matrix whose $(n, k)$ th entry is given by $\mathbf{F}_{n, k}=\frac{1}{\sqrt{N}} e^{-j \frac{2 \pi k n}{N}} ; 0 \leq n, k \leq N-1$. A subscript $t\left(\mathbf{a}_{t}\right)$ denotes a time domain vector. Frequency domain vectors have no subscript. If $\mathbf{a}=\mathbf{F} \mathbf{a}_{t}$, then $\mathbf{a}^{\#}=\mathbf{F a}_{t}^{*}$. Similarly if $\mathbf{B}=\mathbf{F A} \mathbf{F}^{H}$, then $\mathbf{B}^{\#}=\mathbf{F} \mathbf{A}^{*} \mathbf{F}^{H}$. For a matrix $\mathbf{A}, \operatorname{diag}(\mathbf{A})$ denotes the vector of its diagonal elements. $\operatorname{tr}(\mathbf{A})$ is the trace of matrix $\mathbf{A} . \mathbf{I}_{k}$ is the identity matrix of size $k$. 


\section{Model And Assumptions}

\section{A. I/Q Imbalance Only}

Ideally, the I(In-phase) and Q(Quadrature) branches of a receiver should output sinusoids of the same amplitude and with exactly $90^{\circ}$ phase difference. When this requirement is not met, the resulting impairment is called $\mathrm{I} / \mathrm{Q}$ imbalance. It can be modeled as follows [3]

$$
z(t)=\mu y(t)+\nu y^{*}(t)
$$

where $z(t)$ and $y(t)$ are, respectively, the time-domain received signals before and after IQ-imbalance. The scalars $\mu$ and $\nu$ are related to the actual $\mathrm{I} / \mathrm{Q}$ imbalance parameters $\alpha$ (amplitude) and $\theta$ (phase) as follows

$$
\begin{aligned}
& \mu=\cos \left(\frac{\theta}{2}\right)+j \alpha \sin \left(\frac{\theta}{2}\right) \\
& \nu=\alpha \cos \left(\frac{\theta}{2}\right)-j \sin \left(\frac{\theta}{2}\right) .
\end{aligned}
$$

After sampling at the receiver, (1) can be written as an $N$ dimensional vector of time-domain samples

$$
\mathbf{z}_{t}=\mu \mathbf{y}_{t}+\nu \mathbf{y}_{t}^{*}
$$

The output vector $\mathbf{y}_{t}$ is related to the transmitted vector $\mathbf{x}_{t}$ through a Finite Impulse Response (FIR) filter channel $\mathbf{h}=\left[h_{1} h_{2} \cdots h_{L+1}\right]^{T}$ (with memory $L$ ) and noise vector $\mathbf{v}_{t}$ as follows

$$
\mathbf{y}_{t}=\mathbf{H} \mathbf{x}_{t}+\mathbf{v}_{t}
$$

where $\mathbf{v}_{t}$ is Additive White Gaussian Noise (AWGN) vector with co-variance matrix $\sigma_{v}^{2} I_{N}$. The circulant convolution matrix $\mathbf{H}$ is constructed from the channel tap vector $\mathbf{h}$. Since $\mathbf{H}$ is circulant, it can be decomposed in terms of the DFT matrix $\mathbf{F}$ as $\mathbf{F}^{H} \mathbf{G F}$ where $\mathbf{G}$ is a diagonal matrix with elements diag $(\mathbf{G})=\left[g_{1} \cdots g_{N}\right]^{T}$ equal to the DFT of $\mathbf{h}$. Hence

$$
\mathbf{y}_{t}=\mathbf{F}^{H} \mathbf{G F} \mathbf{x}_{t}+\mathbf{v}_{t}
$$

Multiplying both sides of (4) by $\mathbf{F}$ and defining $\mathbf{F y}_{t}=\mathbf{y}$, $\mathbf{F} \mathbf{x}_{t}=\mathbf{x}$ and $\mathbf{F} \mathbf{v}_{t}=\mathbf{v}$ as the corresponding DFT vectors, we have

$$
\mathbf{y}=\mathbf{G x}+\mathbf{v}
$$

Similarly, for $\mathbf{y}_{t}^{*}$, we can show that

$$
\mathbf{y}^{\#}=\mathbf{G}^{\#} \mathbf{x}^{\#}+\mathbf{v}^{\#}
$$

If $\mathbf{x}=\left[x_{1} x_{2} \cdots x_{\frac{N}{2}-1} x_{\frac{N}{2}} x_{\frac{N}{2}+1} \cdots x_{N}\right]^{T}$, then $\mathbf{x}^{\#}=$ $\left[x_{1} x_{N} \cdots x_{\frac{N}{2}+1} x_{\frac{N}{2}} x_{\frac{N}{2}-1} \cdots, x_{2}\right]^{2}$, i.e. the elements of $\mathbf{x}$ are conjugated and reflected about the $\frac{N}{2}$ element excluding the first one ${ }^{1}$. The reason for this rearrangement is shown in

\footnotetext{
${ }^{1}$ This assumes the size of $\mathbf{x}$ to be even which is the case in practice since the DFT size is chosen to be a power of 2 for efficient implementation using the FFT.
}

Appendix I. Moreover, since $\mathbf{G}$ is a diagonal matrix, $\mathbf{G}^{\#}$ is obtained by similar rearrangement of its diagonal elements. Applying the DFT to (2), we get

$$
\mathbf{z}=\mu \mathbf{y}+\nu \mathbf{y}^{\#}
$$

Substituting (5) and (6) in (7) and carefully rearranging the elements, we get the following $\left(\frac{N}{2}-1\right)$ sub-systems [5]

$$
\tilde{\mathbf{z}}_{k}=\tilde{\mathbf{G}}_{k} \tilde{\mathbf{x}}_{k}+\tilde{\mathbf{v}}_{k} \quad ; 2 \leq k \leq \frac{N}{2}
$$

where $\tilde{\mathbf{z}}_{k}=\left[\begin{array}{c}z_{k} \\ z_{N-k+2}^{*}\end{array}\right], \tilde{\mathbf{x}}_{k}=\left[\begin{array}{c}x_{k} \\ x_{N-k+2}^{*}\end{array}\right]$ and $\tilde{\mathbf{G}}_{k}=$ $\left[\begin{array}{cc}\mu g_{k} & \nu g_{N-k+2}^{*} \\ \nu^{*} g_{k} & \mu^{*} g_{N-k+2}^{*}\end{array}\right]$. The first and $\left(\frac{N}{2}+1\right)$ sub-carriers are not used in practice because the former would usually be among the guard sub-carriers and the latter is the DC one.

\section{B. Mobility Only}

When the user terminal moves at high speeds, the channel can no longer be modeled as a time-invariant FIR system. Rather, we would have the taps evolving with time. At time $k$, the channel taps are $\mathbf{h}_{k}=\left[h_{k, 1} h_{k, 2} \cdots h_{k, L+1}\right]^{T}$ and the convolution matrix $\mathbf{H}$ would no longer be circulant. In the frequency domain, this results in dispersion of symbol energy to adjacent sub-carriers. This is evident in the DFT of $\mathbf{H}$ where we no longer have a diagonal matrix but an approximately banded one as shown below

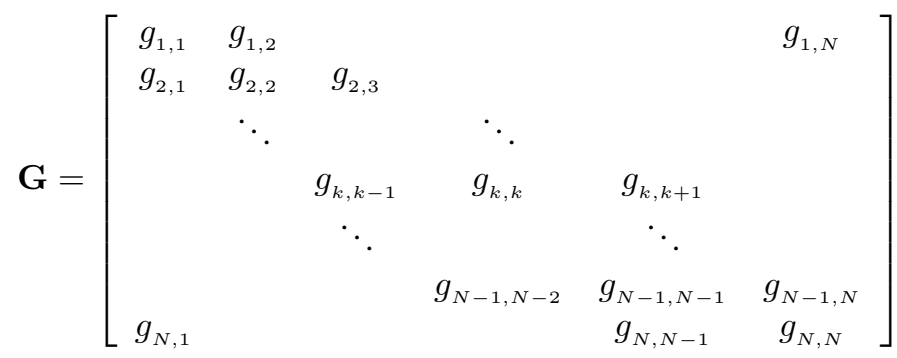

For Doppler rates up to $20 \%$ of sub-carrier bandwidth, three diagonals are sufficient [7] to capture the ICI effect due to mobility. Also, this tri-diagonal approximation reduces the receiver processing complexity. If the above approximation for $\mathbf{G}$ is applied in (5), each element of $\mathbf{y}$ would have the form

$$
y_{k}=g_{k, k-1} x_{k-1}+g_{k, k} x_{k}+g_{k, k+1} x_{k+1}+v_{k}
$$

Therefore, a single-tap Frequency Domain Equalizer (FEQ) is no longer optimum. We will consider a 3-tap FEQ as a performance-complexity tradeoff as suggested in [7]. Although we assumed that the 3 main diagonals of $\mathrm{G}$ are sufficient to capture the ICI effects of mobility, our analysis framework directly applies to the general case where $Q>3$ main diagonals are needed. 


\section{I/Q Imbalance and Mobility}

Modeling I/Q imbalance together with mobility follows the approach of Section II-B except that each of the scalar elements in $\mathbf{G}$ is now a $2 \times 2$ matrix with the same general form as $\tilde{\mathbf{G}}_{k}$ in (8). The first step is to determine $\mathbf{G}^{\#}$ which again is a rearrangement of elements in the diagonals of G. By going through the arguments in Appendix I, it can be shown that

$$
\mathbf{G}^{\#}=\left[\begin{array}{cccccc}
g_{1,1}^{*} & g_{1, N}^{*} & & & & g_{1,2}^{*} \\
g_{N, 1}^{*} & g_{N, N}^{*} & g_{N, N-1}^{*} & & & \\
& \ddots & & \ddots & & \\
& & g_{k, k+1}^{*} & g_{k, k}^{*} & g_{k, k-1}^{*} & \\
& & \ddots & & \ddots & \\
& & & g_{3,4}^{*} & g_{3,3}^{*} & g_{3,2}^{*} \\
g_{2,1}^{*} & & & & g_{2,3}^{*} & g_{2,2}^{*}
\end{array}\right]
$$

Basically, this form also represents a reflection of elements but more involved than in the diagonal case. Now, to obtain the sub-systems, it should be kept in mind that each subcarrier experiences significant ICI from its two adjacent subcarriers, one image sub-carrier and two adjacent sub-carriers of the image sub-carrier, i.e. a total of 5 other sub-carriers. Substituting the block-banded $\mathbf{G}$ and $\mathbf{G}^{\#}$ into (7) and carefully rearranging using this intuition, we can arrive at $\left(\frac{N}{2}-1\right)$ subsystems, but of the form

$$
\begin{gathered}
\mathbf{z}_{\mathbf{k}}=\mathbf{G}_{k, k-1} \mathbf{x}_{k-1}+\mathbf{G}_{k, k} \mathbf{x}_{k}+\mathbf{G}_{k, k+1} \mathbf{x}_{k+1}+\mathbf{v}_{k} \\
\text { where } 2 \leq k \leq \frac{N}{2}, \quad \mathbf{x}_{\mathbf{k}}=\left[\begin{array}{c}
x_{k} \\
x_{N-k+2}^{*}
\end{array}\right] \\
\mathbf{G}_{k, l}=\left[\begin{array}{cc}
\mu g_{k, l} & \nu g_{N-k+2, N-l+2}^{*} \\
\nu^{*} g_{k, l} & \mu^{*} g_{N-k+2, N-l+2}^{*}
\end{array}\right] .
\end{gathered}
$$
and

It is clear that (10) is a vectorized form of (9). Moreover, if we ignore the adjacent interference components $\mathbf{x}_{k-1}$ and $\mathbf{x}_{k+1}$ due to mobility in (10), we would obtain (8) and when there is no $\mathrm{I} / \mathrm{Q}$ imbalance (setting $\mu=1$ and $\nu=0$ ), (10) reduces to (9). Hence, (10) models effects due to both I/Q imbalance and mobility.

\section{Digital BASEband Compensation}

We consider an FIR Vector (V)-MMSE-FEQ structure with 3 two-dimensional taps per sub-carrier pair (or effectively 3taps per sub-carrier). To compute the optimum tap settings, we define the following quantities based on (10)

$$
\begin{array}{r}
\overline{\mathbf{z}}_{k}=\left[\begin{array}{c}
\mathbf{z}_{k-1} \\
\mathbf{z}_{k} \\
\mathbf{z}_{k+1}
\end{array}\right], \overline{\mathbf{x}}_{k}=\left[\begin{array}{c}
\mathbf{x}_{k-2} \\
\mathbf{x}_{k-1} \\
\mathbf{x}_{k} \\
\mathbf{x}_{k+1} \\
\mathbf{x}_{k+2}
\end{array}\right], \overline{\mathbf{v}}_{k}=\left[\begin{array}{c}
\mathbf{v}_{k-1} \\
\mathbf{v}_{k} \\
\mathbf{v}_{k+1}
\end{array}\right] \\
\overline{\mathbf{G}}_{k}=\left[\begin{array}{ccccc}
\mathbf{G}_{k-1, k-2} & \mathbf{G}_{k-1, k-1} & \mathbf{G}_{k-1, k} & \mathbf{0} & \mathbf{0} \\
\mathbf{0} & \mathbf{G}_{k, k-1} & \mathbf{G}_{k, k} & \mathbf{G}_{k, k+1} & \mathbf{0} \\
\mathbf{0} & \mathbf{0} & \mathbf{G}_{k+1, k} & \mathbf{G}_{k+1, k+1} & \mathbf{G}_{k+1, k+2}
\end{array}\right]
\end{array}
$$

Therefore, we have

$$
\overline{\mathbf{z}}_{k}=\overline{\mathbf{G}}_{k} \overline{\mathbf{x}}_{k}+\overline{\mathbf{v}}_{k}
$$

The MMSE estimate for $\tilde{\mathbf{x}}_{k}$ is given by

$$
\hat{\tilde{\mathbf{x}}}_{k}=\mathbf{w}_{k}^{H} \overline{\mathbf{z}}_{k}
$$

where

$$
\mathbf{w}_{k}^{H}=\overline{\mathbf{g}}_{k}^{H}\left(\overline{\mathbf{G}}_{k} \overline{\mathbf{G}}_{k}^{H}+\sigma_{v}^{2} \mathbf{I}_{6}\right)^{-1}
$$

with $\overline{\mathbf{g}}_{k}$ being the middle block column of $\overline{\mathbf{G}}_{k}$. Note that using (13), we detect the $k$-th sub-carrier $x_{k}$ and its image $x_{N-k+2}^{*}$ jointly.

\section{Channel Estimation Scheme}

\section{A. I/Q Imbalance Only}

When there is no ICI, channel estimation involves determining the $\tilde{\mathbf{G}}_{k}$ 's in (8). Towards this goal, we rewrite (8) as suggested in [5] in the following form

$$
\tilde{\mathbf{z}}_{k}=\left[\begin{array}{cccc}
s_{k} & 0 & s_{N-k+2}^{*} & 0 \\
0 & s_{k} & 0 & s_{N-k+2}^{*}
\end{array}\right]\left[\begin{array}{c}
\mu g_{k} \\
\nu^{*} g_{k} \\
\nu g_{N-k+2}^{*} \\
\mu^{*} g_{N-k+2}^{*}
\end{array}\right]+\tilde{\mathbf{v}}_{k}
$$

where $\mathbf{s}_{k}$ 's are the training pilots. Equation (15) is an underdetermined system of equations and we need two more equations to solve it. To do this, we need another sub-carrier that experiences approximately the same channel. Therefore, we assign pilots in two adjacent sub-carriers and each such pair has a corresponding pilot pair image. The preamble has a similar placement of training pilots, only that it has a denser packing. This pattern is shown in Figure 1. For a delay spread $\tau \leq \frac{2}{T}$ (where $T$ is the OFDM symbol duration), the channel for the $k$ and $(k+1)$ sub-carriers would approximately be the same. Similarly, the corresponding image sub-carrier pair with indices $N-k+2$ and $N-k+1$ would also roughly have the same channel response. Using this fact and (15), we can write

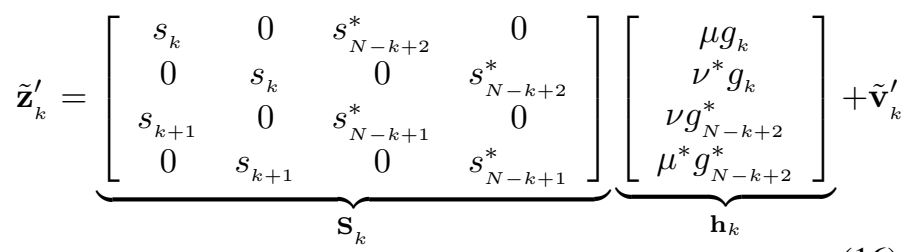

where $\tilde{\mathbf{z}}_{k}^{\prime}=\left[\begin{array}{c}\tilde{\mathbf{z}}_{k} \\ \tilde{\mathbf{z}}_{k+1}\end{array}\right], \tilde{\mathbf{v}}_{k}^{\prime}=\left[\begin{array}{c}\tilde{\mathbf{v}}_{k} \\ \tilde{\mathbf{v}}_{k+1}\end{array}\right], g_{k}$ is the channel common to the sub-carrier pair $(k, k+1)$ and $g_{N-k+2}$ to the subcarrier pair $(N-k+1, N-k+2)$. Let $\mathcal{S}_{k}=\left[\begin{array}{cc}s_{k} & s_{N-k+2}^{*} \\ s_{k+1} & s_{N-k+1}^{*}\end{array}\right]$, which is nothing but a condensed form of $\mathbf{S}_{k}$ obtained by eliminating the zero elements. To achieve the lowest channel estimation MSE, $\mathcal{S}_{k}$ should be orthogonal . Under the restriction of binary pilots, this could be easily achieved by choosing 


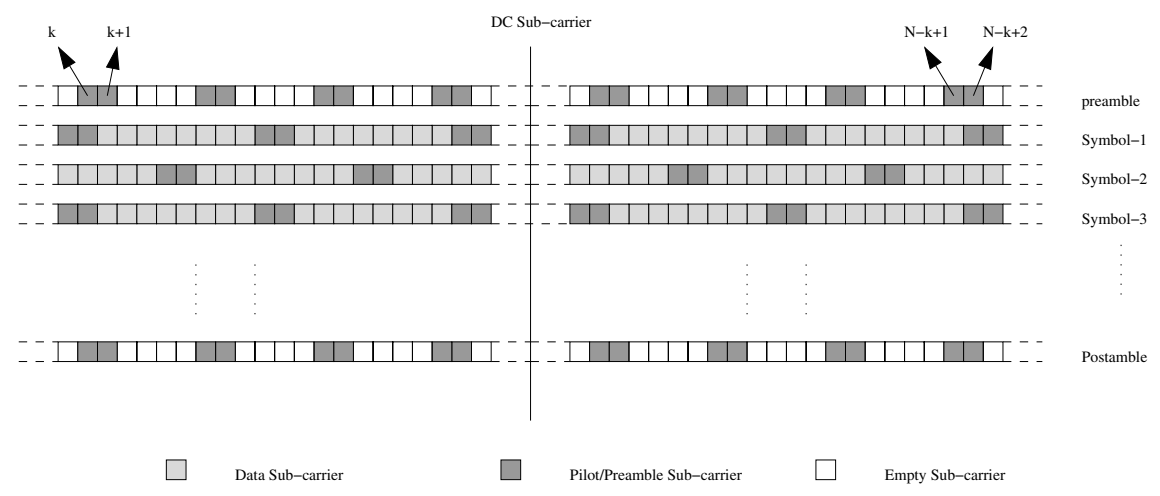

Fig. 1. Preamble and Pilot Pattern

$\mathcal{S}_{k}$ to be the Hadamard matrix $\left[\begin{array}{cc}1 & 1 \\ 1 & -1\end{array}\right]$. The linear Least Squares (LS) estimate of $\mathbf{h}_{k}$ is

$$
\hat{\mathbf{h}}_{k}=\mathbf{S}_{k}^{-1} \tilde{\mathbf{z}}_{k}^{\prime}
$$

since $\mathbf{S}_{k}$ is square. The estimation MSE is given by

$$
\sigma_{c e}^{2}=\sigma_{v}^{2} \operatorname{tr}\left(\left(\mathbf{S}_{k}^{H} \mathbf{S}_{k}\right)^{-1}\right)
$$

Given the structure of $\mathbf{S}_{k}$, we can easily show that $\sigma_{c e}^{2}=$ $2 \frac{\sigma_{v}^{2}}{E_{p}}$, where $E_{p}$ is the energy of the pilot tone. It is common to boost the energy in pilot tones and hence the MSE could be kept small.

\section{B. Mobility Only}

Under high mobility, each of the channel taps of $\mathbf{h}_{k}$ as modeled in Section II-B varies within each OFDM symbol. In [9], it was shown that the taps could be accurately approximated to be linearly varying for a normalized Doppler of up to $20 \%$. Let $\mathbf{h}_{k}^{(i)}$ represent the taps of OFDM symbol $i$. Following [9], we can model the $k$-th sample of $l$-th tap as

$h_{k, l}^{(i)}=\bar{h}_{l}^{(i)}+\alpha_{l}^{(i)}\left(k+1-\frac{N}{2}\right) \quad 0 \leq k \leq N-1,0 \leq l \leq L$

where $\bar{h}_{l}^{(i)}$ denotes the average value of the $l$-th tap in symbol $i$. The best approximation is achieved when $\bar{h}_{l}^{(i)}$ is chosen to be equal to $h_{\frac{N}{2}, l}^{(i)}$. The set of mid-symbol tap weights $\left\{h_{\frac{N}{2}, l}^{(i)}, 0 \leq\right.$ $l \leq L\}$ is related to the diagonal of the banded matrix $\mathbf{G}$ through a DFT. Ignoring ICI effects, the diagonal of $\mathbf{G}$ could be estimated using pilots.

To determine the slope $\alpha_{l}^{(i)}$, [9] proposes a scheme which involves the $(i-1)$ and $(i+1)$ OFDM symbols. The main idea is that the slope remains constant for one OFDM symbol duration. This duration can be chosen from the mid-point of one symbol to the mid-point of the next one. Since the mid-symbol tap weights $h_{l}^{(i)}$ could be estimated as described earlier, the tap weights for all other time instants $k$ can be obtained using (19). Now, OFDM symbol $i$ would not have a single slope $\alpha_{l}^{(i)}$ for tap $l$, rather would have a slope $\alpha_{l}^{(i-1, i)}=\left(\bar{h}_{l}^{(i)}-\bar{h}_{l}^{(i-1)}\right) / N$ for the first half and a slope $\alpha_{l}^{(i, i+1)}=\left(\bar{h}_{l}^{(i+1)}-\bar{h}_{l}^{(i)}\right) / N$ for the second half. It should be noted that every useful OFDM symbol containing data needs to be preceded and succeeded by other symbols for this kind of interpolation. To ensure this, we insert a preamble before and a postamble after the first and last OFDM symbols of the frame, respectively, as shown in Figure 1. To avoid this overhead, one can also adopt the cyclic prefix based scheme described in [9] for slope estimation.

\section{IQ-Imbalance and Mobility}

In the presence of both I/Q Imbalance and mobility, we first estimate the diagonals of $\mu \mathbf{G}$ and $\nu \mathbf{G}^{\#}$ using the method described in Section IV-A as if there is no ICI. This assumption is valid because the energy of the main diagonal is much larger than the energy of the other diagonals in $\mathbf{G}$ and $\mathbf{G}^{\#}$. Moreover, pilots are generally boosted in comparison to data. This would give us the $\mu g_{k, k}$ and $\nu g_{k, k}^{*}$ estimates. Applying the procedure described in Section IV-B to $\mu \mathbf{G}$ and $\nu \mathbf{G}^{\#}$ separately, we can obtain their off-diagonal elements in both cases. Finally, the $\tilde{\mathbf{G}}_{k}$ 's of Equation (11) could be formed from the estimated $\mu \mathbf{G}$ and $\nu \mathbf{G}^{\#}$.

\section{COMPleXity ANALYSis}

For a 512 sub-carrier OFDM system, we analyze the complexity of the major steps involved. With $L=16$, using the method described in [7], the number of complex multiplications required to compute $\mu \mathbf{G}$ would be 2848 . This holds also for the computation of $\nu \mathbf{G}^{\#}$. To obtain the V-MMSE-FEQ tap weights, we would need to do 256 matrix inversions, each of size $6 \times 6$. This would amount to 9216 complex multiplications. Finally, the equalization step would cost us 3072 complex multiplications. Therefore, one OFDM block would require a total of 17,984 complex multiplications. The 512 sub-carrier mode of 802.16 has a symbol duration of $94.28 \mu \mathrm{s}$. This would imply about 190 Million Instruction Per Second (MIPS).

\section{Simulation Results}

The OFDM system we simulate is similar to the 512subcarrier profile of the $802.16 \mathrm{e}$ mobile WiMAX standard. The 


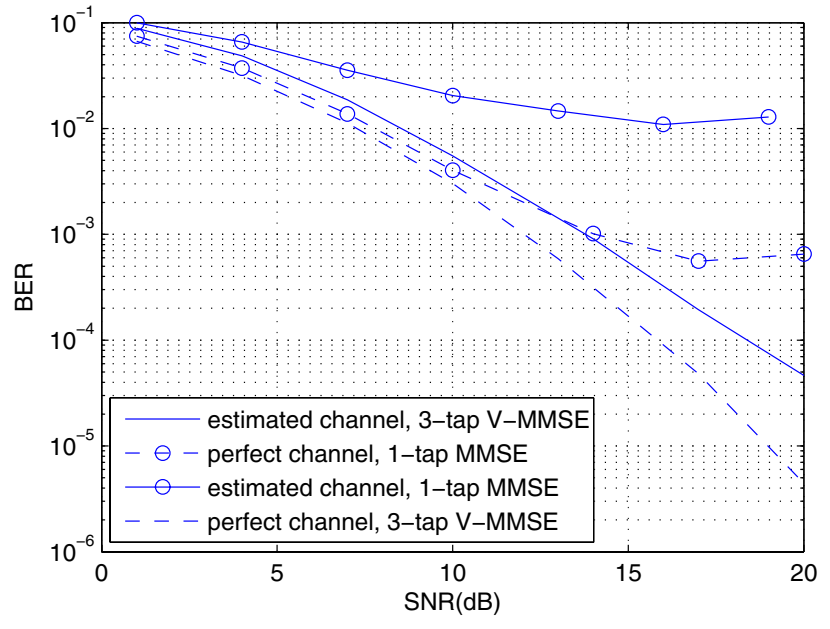

Fig. 2. Performance gains with 3-tap V-MMSE FEQ with ideal and estimated channel information in the presence of $10 \%$ Doppler and I/Q Imbalance with $1 \mathrm{~dB}$ and $2^{\circ}$.

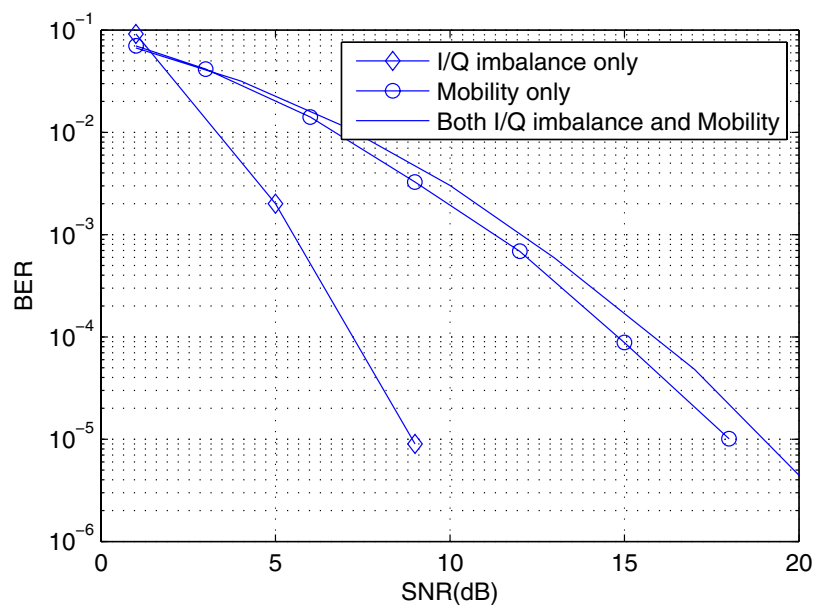

Fig. 3. Performance comparison of digital baseband compensation under I/Q Imbalance only, mobility only and both

sampling frequency and the number of guard and pilot subcarriers we use are as specified in the standard. This amounts to 45 guard carriers on the left, 46 on the right, 360 data and 60 pilot sub-carriers. Channel coding is the rate- $\frac{1}{2}$ Tail Biting Convolutional Code (TBCC) with interleaving as specified in the standard. We differ from the standard in the location and assignment scheme of the pilots and preamble. The channel's power delay profile is that of Stanford University Interim (SUI3) specification with mobility according to Jakes model.

In the first experiment, we study the performance gains due to the proposed V-MMSE-FEQ scheme under perfect and estimated channel information. We assume IQ imbalance parameters of $\left(\alpha=1 \mathrm{~dB}, \theta=2^{\circ}\right)$ and a normalized Doppler of $10 \%$. Figure 2 shows the results where we can see that in the conventional 1-tap FEQ, there is always an error floor that limits performance, and is eliminated by using our V-MMSE-
FEQ. In the second experiment, we compare the performances of the impairments individually and combined. When there is only I/Q imbalance $\left(\alpha=1 \mathrm{~dB}, \theta=2^{\circ}\right)$, we use 1 -tap V-MMSE equalization. When there is only mobility $(10 \%$ normalized Doppler), we use a 3-tap MMSE FEQ. When both impairments are present, we use the 3-tap V-MMSE equalization as in the previous experiment. With perfect channel information, the results are shown in Figure 3. Under the assumed parameters, mobility limits the performance and our proposed compensation scheme approaches this limit to within $1 \mathrm{~dB}$.

\section{CONCLUSions}

In this paper, we developed a unified OFDM ICI frequency domain model for I/Q imbalance and mobility. The model is a 3-tap FIR filter where each tap is a $2 \times 2$ matrix whose elements depend on the channel frequency response and the I/Q imbalance parameters. To compensate for this ICI, we proposed a vector-MMSE-based digital baseband compensation scheme. Also we proposed an efficient pilot allocation strategy and channel estimation scheme. Our simulation results clearly show that our proposed compensation scheme using 3 taps per sub-carrier achieves significant performance gains. We emphasize that our scheme does not require offline knowledge of the I/Q imbalance model parameters which is a significant implementation advantage. Moreover, our approach can easily accommodate the case of frequency-dependent I/Q imbalance.

\section{APPENDIX I \\ DFT OF CONJUGATED MATRICES}

Let $\mathbf{G}$ be the DFT of $\mathbf{H}$ and $\mathbf{G}^{\#}$ that of $\mathbf{H}^{*}$.

$$
\begin{aligned}
\mathbf{G} & =\mathbf{F H} \mathbf{F}^{H} \\
\mathbf{G}^{\#} & =\mathbf{F H}^{*} \mathbf{F}^{H}=\left(\mathbf{F}^{*} \mathbf{H}\left(\mathbf{F}^{H}\right)^{*}\right)^{*} \\
& =(\mathbf{F}^{*} \mathbf{F}^{H} \underbrace{\mathbf{F H} \mathbf{F}^{H}}_{\mathbf{G}} \mathbf{F}\left(\mathbf{F}^{H}\right)^{*})^{*} \\
& =\left(\mathbf{F}^{*} \mathbf{F}^{H} \mathbf{G} \mathbf{F}\left(\mathbf{F}^{H}\right)^{*}\right)^{*} \\
& =\mathbf{F F}^{\mathbf{T}} \mathbf{G}^{*} \mathbf{F}^{*} \mathbf{F}^{H}=\mathbf{F F}^{\mathbf{T}} \mathbf{G}^{*}\left(\mathbf{F F}^{\mathbf{T}}\right)^{*}
\end{aligned}
$$

It can be easily checked that $\mathbf{F F}^{\mathbf{T}}$ is a permutation matrix

$$
\mathbf{P}=\mathbf{F F}^{\mathbf{T}}=\left[\begin{array}{cccccc}
1 & 0 & \ldots & 0 & 0 & 0 \\
0 & 0 & \ldots & 0 & 0 & 1 \\
0 & 0 & \ldots & 0 & 1 & 0 \\
& \ldots & \ldots & \ldots & & \\
& \ldots & \ldots & \ldots & & \\
0 & 1 & \ldots & 0 & 0 & 0
\end{array}\right]
$$

Pre-multiplying $\mathbf{G}^{*}$ with $\mathbf{P}$ results in reflection of its rows about the $\left(\frac{N}{2}+1\right)$ row with the first row not being affected. Post-multiplying $\mathbf{G}^{*}$ with $\mathbf{P}$ results in reflection of its columns about the $\left(\frac{N}{2}+1\right)$ column with the first column not being affected. 


\section{REFERENCES}

[1] G. Fettweis, M. Lohning, D. Petrovic, M. Windisch, P. Zillmann, W. Rave, "Dirty RF: a new paradigm", 2005 IEEE 16th International Symposium on Personal, Indoor and Mobile Radio Communications Volume 4, 11-14 Sept. 2005 Page(s):2347 - 2355

[2] B. Razavi,"RF Microelectronics”, Englewood Cliff, NJ: Prentice-Hall, 1998

[3] C. L. Liu, "Impacts of I/Q imbalance on QPSK-OFDM-QAM detection", IEEE Trans. Consum. Electron., vol. 44, no. 3, pp. 984-989, Aug. 1998

[4] A. Schuchert, R. Hasholzne and P. Anotine, "A novel IQ imbalance compensation scheme for the reception of OFDM signals", IEEE Trans. Consum. Electron., vol. 47, no. 3, pp. 313-318, Aug. 2001

[5] A. Tarighat, R. Bagheri, A. H. Sayeed, "Compensation schemes and peformance analysis of IQ imbalances in OFDM receivers", IEEE Trans. Signal Process., vol. 53, no. 8, pp. 3257-3268, Aug. 2005

[6] S. Kim and G. J. Pottie, "Robust OFDM in fast fading channel", Globecom, vol. 2, pp. 1074-1078, Dec. 2003

[7] S. Lu, R. Kalbasi, and N. Al-Dhahir, "OFDM interference mitigation algorithms for doubly-selective channels," In Proc. of IEEE Vehicular Tenology Conference, Sep 2006.

[8] Mikko Valkama, Marku Renfors and Visa Koivunen,"Blind I/Q Imbalance Compensation in OFDM Receivers Based on Adaptive I/Q Signal Decorrelation", ISCAS(3) 2005, pp. 2611-2614

[9] Y. Mostofi and D. C. Cox, "ICI mitigation for pilot-aided OFDM mobile systems", IEEE Trans. Wireless Commun., vol. 4, pp. 765-774, Mar. 2005 